\title{
Prática Avançada em Enfermagem na Saúde da Mulher: formação em Mestrado Profissional
} Women's Health Care in Advanced Practice Nursing: a professional master's degree program Práctica Avanzada de Enfermería en Salud de la Mujer: formación en Maestría Profesional

Cibele Andruccioli de Mattos-Pimenta ${ }^{1}$ io https://orcid.org/0000-0003-3624-6806

Kelly Pereira Coca ${ }^{1}$ id https://orcid.org/0000-0002-3604-852X

Maria Helena Costa Amorim ${ }^{1}$ io https://orcid.org/0000-0002-4252-7092 Angélica Gonçalves Silva Belasco ${ }^{1}$ in nttps://orcid.org/0000-0002-0307-6225

Maria Cristina Gabrielloni ${ }^{1}$ io https://orcid.org/0000-0003-2395-9161

Janine Schirmer ${ }^{1}$ io https://orcid.org/0000-0003-0783-2961

\section{Como citar:}

Mattos-Pimenta CA, Coca KP, Amorim MH,

Belasco AG, Gabrielloni MC, Schirmer J.

Prática Avançada em Enfermagem na Saúde

da Mulher: formação em Mestrado Profissional.

Acta Paul Enferm. 2020;33:eAPE20200123.

DOI

http://dx.doi.org/10.37689/actaape/2020AE01235

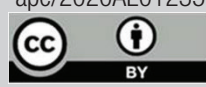

Descritores

denfermagem; Saúde da mulher; Educação em enfermagem; Capacitação de recursos humanos em saúde; Educação de pós-graduação em enfermagem

Keywords

Advanced practice nursing; Women's health Education, nursing; Health human resource training; Education, nursing, graduate

Descriptores

Enfermería de práctica avanzada; Salud de la mujer; Educación en enfermería; Capacitación de recursos humanos en salud; Educación de postgrado en enfermería

Submetido 1 de Junho de 2020

Aceito

20 de Agosto de 2020

Autor correspondente

Maria Helena Costa Amorim

E-mail: mhcamorimap@gmail.com

\section{Resumo}

0 Mestrado Profissional em Prática Avançada na Saúde da Mulher integrou os conceitos de Enfermagem em Prática Avançada ao Mestrado Profissional. As Enfermeiras de Prática Avançada desenvolvem competências clínicas e de pesquisa superiores às tradicionais e desempenham papéis visando a melhoria contínua de resultados em saúde, a inclusão de mais pessoas no sistema de saúde e a expansão dos limites da prática. As Enfermeiras de Prática Avançada atuam na inovação, na construção e na implementação de protocolos, de processos de cuidado institucionais e de políticas públicas. Três focos compõem o programa: 1) Aumentar a autonomia da enfermeira obstetra para a tomada de decisão no pré-natal, no parto e no pós-parto, especialmente em domicílio, casas e centros de parto; 2) Impulsionar a competência para o cuidado e a construção de políticas sobre violência contra a mulher; e 3) Propor uma atuação transformadora sobre a saúde mental e reprodutiva da mulher, opção sexual e de gênero. Trata-se de programa inovador que se alinha ao movimento para a introdução da Enfermagem Prática Avançada na América Latina.

\section{Abstract}

The Professional Master's Program Women's Health in Advanced Practice Nursing has integrated the concepts of advanced practice nursing into a professional master's education. Advanced practice nurses develop further clinical and research competencies than traditional nurses, and their roles include continuous improvement of health care outcomes, inclusion of more individuals in the health care system, and expansion the limits for their practice. Advanced practice nurses play a role on promote innovative measures, create and implement protocols, institutional care processes, and public policies. The program focus on the following three areas: 1) To increase obstetric nurse and midwife's decision-making autonomy in antenatal, natal, and postnatal settings, mainly in the context of home birth and birthing centers; 2) To promote care competencies and creation of policies that cover violence against women; and 3) To propose a transformative practice on women's mental and reproductive health, sexual orientation, and gender identity. This innovative Program is aligned with the movement towards the introduction of Advanced Practice Nursing in Latin America.

\section{Resumen}

La Maestría Profesional en Práctica Avanzada en Salud de la Mujer integró los conceptos de Enfermería de Práctica Avanzada a la Maestría Profesional. Las Enfermeras de Práctica Avanzada desarrollan competencias clínicas y de investigación superiores a las tradicionales y desempeñan papeles que buscan la mejora continua de resultados en salud, la inclusión de más personas en el sistema de salud y la expansión de los límites de la práctica. Las Enfermeras de Práctica Avanzada actúan en la innovación, en la elaboración y en la implementación de protocolos, de procesos de cuidado institucionales y de políticas públicas. El programa 
tiene tres focos: 1) aumentar la autonomía de la enfermera obstetra para la toma de decisiones en la atención prenatal, en el parto y en el posparto, especialmente en atención domiciliaria, casas y centros de parto; 2) impulsar la competencia para el cuidado y la elaboración de políticas sobre violencia contra la mujer; y 3) proponer una actuación transformadora sobre la salud mental y reproductiva de la mujer, opción sexual y de género. Se trata de un programa innovador, que está en línea con el movimiento para la introducción de la Enfermería de Práctica Avanzada en América Latina.

\section{Introdução}

A enfermagem como "espinha dorsal” e sustentáculo do sistema de saúde é tradicional e conhecida em todo o mundo. No entanto, a enfermeira com atuação mais autônoma, como propositora de políticas e ações transformadoras da realidade em saúde, como empreendedora de soluçóes criativas e tecnológicas capazes de modificar positivamente resultados em saúde e como pesquisadora para solucionar os desafios do cuidado avançado é pouco conhecida no Brasil e na maior parte dos países em desenvolvimento. Profissionais com essas competências avançadas estão presentes em países de primeiro mundo e têm modificado a ciência e os resultados dos cuidados em saúde e em enfermagem. Não os ter deixa em desvantagem nosso sistema de saúde.

Estudo patrocinado pelo grupo de parlamentares do Reino Unido, All Party-Parliamentary Group on Global Health (APPG) em busca da melhoria do cuidado em saúde demonstrou que potencializar a atuação das enfermeiras por meio do fortalecimento e da ampliação de suas competências pode melhorar a saúde, promover a igualdade de gênero e auxiliar no crescimento econômico. O relatório da APPG's Triple Impact of Nursing (2016) afirma que, sem fortalecer o papel das enfermeiras, não é possível alcançar saúde universal. Essas conclusóes e recomendações foram enviadas para o Governo do Reino Unido, à Uniâo Européia e à Organização Mundial de Saúde (OMS) e foram tão bem recebidas que decidiu-se lançar uma campanha global denominada Nursing Now, em parceria com a OMS e o International Council of Nursing (ICN). A campanha Nursing Now é de três anos (2018-2020) e visa melhorar a saúde globalmente, elevando o status e a educação da enfermagem e da enfermeira. A campanha conseguiu adeptos em todo o mundo. Vários países fizeram investimentos na enfermagem e 2020 foi designado como o Ano da Enfermeira e da Enfermeira Obstetra. ${ }^{(1)}$

No Brasil, a formação e a capacitação do enfermeiro dão-se por diversos modos: bacharelado, especializaçóes $(360 \mathrm{~h})$, programas de residência (5.760h), busca autodidata para aquisição de competências por meio de cursos breves, congressos, estudos independentes e programas de mestrado e doutorado acadêmicos e profissionais, estes últimos ainda incipientes. $\mathrm{O}$ mestrado e o doutorado acadêmicos, embora consolidados em nosso meio, visam à formação de professores e pesquisadores, enquanto o mestrado profissional (MP) visa aperfeiçoar profissionais para atuar no mundo do trabalho, de modo reflexivo, crítico e transformador. ${ }^{(2)}$

A variedade de caminhos para adquirir mais competências tem permitido a qualificação de parcela dos enfermeiros, mas a formação pode ser mais morosa, menos eficiente, muito restrita e não resultar no aperfeiçoamento do cuidado em enfermagem e saúde na velocidade e consistência que se necessita, comparada à formação por programas estruturados que visam implementar no mercado de trabalho profissionais altamente diferenciados para transformação e aperfeiçoamento do cuidado prestado.

A capacitação mais orientada para a clínica avançada foi instituída na década de 1980 em países desenvolvidos como Canadá, EUA, Inglaterra, Austrália e outros, sob a denominação de Enfermeiro de Prática Avançada (EPA) e tem se mostrado efetiva para a inclusão de profissionais com competências específicas e diferenciadas no sistema de saúde, a melhora dos cuidados e dos indicadores de saúde. No Brasil, existe a oportunidade de implantá-la, bem como inúmeros campos de atuação para EPA. Em nosso meio, em decorrência da ausência de padronização para a formação de EPA (competência clínica avançada, capacidade de investigação e liderança diferenciadas), muitas vezes, não há papéis profissionais pré-estabelecidos que os mais qualificados possam exercer, nem remuneração diferenciada ou título que represente adequadamente esses profissionais. $^{(3)}$

EPAs são profissionais com habilidades e competência clínica para tomar decisóes complexas, expandir e aperfeiçoar a prática de enfermagem, pro- 
mover maior inclusão e melhor cuidado na assistência à saúde, dentro do contexto da prática baseada em evidência e da inovaçáo tecnológica. $\mathrm{O}$ grau de mestre é a exigência mínima de formação. ${ }^{(4)}$

EPA é um nível de prática mais do que um tipo de prática. São educadas no nível de mestrado, têm conhecimento e prática clínica de experts e são reconhecidas como tal, têm liberdade e autoridade para atuar e tomar decisóes autônomas na avaliação, no diagnóstico e no tratamento dos doentes. ${ }^{(5)}$

A Organização Panamericana de Saúde (OPAS) e a OMS elaboraram o documento "Ampliação do papel dos enfermeiros na atenção primária à saúde", ${ }^{(3)}$ no qual conclamam os governos e enfermeiros de países da América a implementarem a formação de EPA para a atenção primária e para a área da enfermagem obstétrica, visto o potencial das enfermeiras das Américas Central e do Sul, que já contam com programas de especialização, residência, mestrado e doutorado acadêmicos. Com isso pretende-se dar maior cobertura e assistência aos usuários do sistema de saúde, aproveitar a capacidade intelectual dos enfermeiros e reter bons profissionais na profissão. Para a OPAS, novos perfis profissionais como EPA, além de contribuir para a promoção da saúde, prevenir doenças e reduzir mortes, podem assumir mais funçóes com autonomia nos serviços de atenção primária de zonas vulneráveis em cidades e em áreas remotas. ${ }^{(3)}$

Editorial da revista Lancet, no ano internacional da Enfermagem (2020), ressaltou a importância da profissão, que corresponde a quase metade dos recursos humanos em Saúde no mundo, com cerca de 20 milhôes de enfermeiras e 2 milhôes de parteiras, "Trabalhando em ampla variedade de funçôes e em muitos contextos diferentes, enfermeiros são, com frequência, os primeiros e únicos profissionais de saúde que as pessoas têm acesso". A conceituada Revista científica defende a expansão das clínicas de Enfermagem como forma de permitir "uma expansão rápida e custo-efetiva dos serviços para doenças não transmissíveis, práticas avançadas de enfermagem e enfermeiros especialistas que poderiam fortalecer a atenção primária”, dentre outros setores. ${ }^{(6)}$

O Conselho Federal de Enfermagem (COFEN) tem apoiado a iniciativa de EPA, criou comissão para estudar a implantação desse profissional no Brasil e vem subsidiando Programas de Mestrado Profissional. Considera que enfermeiros com formação em práticas avançadas são fundamentais na promoção da saúde e prevenção de doenças. ${ }^{(7)}$

As necessidades de formação do Enfermeiro de Prática Avançada coadunam-se, perfeitamente, com a formação em mestrado profissional, que visa formação de profissionais que impulsionem a inovação voltada para o mundo do trabalho e tem como finalidade:

I - capacitar profissionais qualificados para o exercício da prática profissional avançada e transformadora de procedimentos, visando atender demandas sociais, organizacionais ou profissionais e do mercado de trabalho; II - transferir conhecimento para a sociedade, atendendo demandas específicas e de arranjos produtivos com vistas ao desenvolvimento nacional, regional ou local; III - promover a articulação integrada da formação profissional com entidades demandantes de naturezas diversas, visando melhorar a eficácia e a eficiência das organizaçôes públicas e privadas por meio da solução de problemas e geração e aplicação de processos de inovação apropriados; IV - contribuir para agregar competitividade e aumentar a produtividade em empresas, organizaçóes públicas e privadas. ${ }^{(8)}$

O mestrado profissional é terminal e encerra-se em si mesmo, visa que o profissional desenvolva habilidades iniciais em pesquisa, que se habitue a consumir pesquisa, aprenda a localizar estudos que interessem à sua profissão, a implementá-los no seu ambiente de trabalho, a medir resultados e a desenvolver e implementar inovaçóes. Tudo isso requer que o aluno desenvolva competências avançadas para o mundo do trabalho. As competências a serem adquiridas no mestrado profissional são diversas, mas tão complexas quanto as do mestrado acadêmico. Os objetivos do mestrado profissional proposto pela CAPES alinham-se perfeitamente à formação de EPA, daí a pertinência de se articular os dois referenciais.

Frente à oportunidade para a instalação do Modelo de EPA no Brasil e o reconhecimento da academia sobre a relevância do mestrado profissional para a capacitação transformadora para o mun- 
do do trabalho, a Escola Paulista de Enfermagem da UNIFESP (EPE-UNIFESP) propôs a organização do Programa de Mestrado Profissional em Prática Avançada em Enfermagem, com a Área de Concentração Prática Avançada de Enfermagem na Saúde da Mulher (MEPASM), com a possibilidade de, no futuro, criar outras áreas de concentração. $\mathrm{O}$ objetivo deste artigo é apresentar essa proposta.

\section{Métodos}

A proposta surgiu do anseio da Escola Paulista de Enfermagem de organizar mestrado profissional em EPA, da existência de corpo docente com grande expertise na área de Saúde da Mulher e pela oportunidade da instituição contar com duas professoras visitantes com experiência em organização de programas de pós-graduação. A proposta baseou-se na literatura sobre EPA, ${ }^{(3,4,5,9-11)}$ sobre mestrado profissional e normativas da CAPES, de reunióes com a Diretoria, Câmara de Pós-Graduação, Comissão de Pós-Graduação, docentes da instituição e docentes da área de Enfermagem na Saúde da Mulher da EPE. Foi avaliada por pares dentro da instituição, pela Câmara de Pós-Graduação da EPE UNIFESP, pela Pró Reitoria de Pós-Graduação da UNIFESP e, só então, submetida à CAPES. A decisão sobre quais seriam os eixos centrais e disciplinas da proposta basearam-se nos preceitos de EPA, na prevalência epidemiológica dos eventos/agravos no Brasil relativos à saúde da mulher, das lacunas e da necessidade de impulsionar cuidado transformador nessas áreas.

\section{Resultados}

A Escola Paulista de Enfermagem conta com docentes e pesquisadores com experiência comprovada na formação de mestres, doutores e em pesquisa. A PósGraduação stricto sensu teve início em 1978, com o Mestrado em Enfermagem Pediátrica, seguindo-se do Mestrado em Enfermagem Obstétrica em 1980, o doutorado em Enfermagem Materna e Infantil em 1986 e Enfermagem na Saúde do Adulto em 1989.
O programa atual consta de Curso de Mestrado, Doutorado e Dinter com a Universidade Federal do Acre. A EPE abriga também o Programa de MP em Ensino em Ciências da Saúde.

O Departamento de Enfermagem na Saúde da Mulher da EPE tem larga tradição e liderança nacional na formação de enfermeiras obstetras desde 1979, por meio de cursos de especialização e residência. A expertise e liderança de suas docentes na comunidade e associaçóes de enfermeiras sustentam uma proposta de mestrado profissional em Práticas Avançadas na Saúde da Mulher.

\section{Objetivos do Programa e perfil do egresso}

Da análise do perfil epidemiológico, das políticas de saúde e carências de cuidado na área de saúde da mulher, dos preceitos de EPA e dos objetivos do MP escolheram-se três grandes temas que constituem os eixos norteadores dessa proposta: maior autonomia da enfermeira na atenção ao pré-natal, parto e puerpério; atuação transformadora da enfermeira de saúde de mulher junto à mulher vítima de violência e nas questóes relacionadas à saúde mental e reprodutiva, opção sexual e de gênero. A partir desses eixos norteadores foram estabelecidos os seguintes objetivos.

- Aprimorar a capacitação de profissionais para cuidado avançado da mulher no ciclo gravídico puerperal, no período neonatal, na saúde sexual e reprodutiva, relacionado às questóes de opção sexual e de gênero e junto às vítimas de violência, visando atuação transformadora e de inovação, consonante às demandas sociais.

- Qualificar profissionais pelo aprendizado rigoroso de métodos e fundamentos científicos para desenvolver estudos inovadores e robustos.

- Desenvolver competências profissionais de atualização e incorporação continuada dos avanços da ciência e das tecnologias na área de saúde da mulher, de produção de ciência aplicada, proposição de inovações e desenvolvimento de tecnologias para a solução de problemas específicos.

A intenção é formar profissionais que tenham competências do EPA na área de atenção à mulher, conforme proposto pelo ICN como: alto grau de 
autonomia profissional e prática independente; habilidades de gerenciamento de casos; habilidades avançadas de avaliação da saúde; habilidades de tomada de decisão, de diagnóstico e raciocínio clínico; reconhecidas competências clínicas avançadas; prestação de serviços de consultoria a prestadores de saúde; implementação de planos e avaliação de programas.

Ao final do mestrado profissional pretende-se que o pós-graduando tenha agregado avanços na sua formação, demonstrando conhecimento crítico, reflexivo e baseado nas melhores evidências na área de saúde da mulher, favorecendo a transformação e a melhoria dos indicadores de saúde e bem-estar; que tenha desenvolvido maior flexibilidade cognitiva, criatividade e pensamento crítico, habilidades especiais de negociação, de julgamento e tomada de decisão, inteligência emocional, competências de gestão de processos, projetos e pessoas; habilidade de síntese e análise dos dados, uso e desenvolvimento de ferramentas tecnológicas já existentes para o cuidado da mulher no âmbito individual e coletivo.

\section{Caracterização do curso: área de concentração, linhas de pesquisa e disciplinas}

O MEPASM foi desenhado para ser uma área de concentração do Mestrado Profissional de Enfermagem em Práticas Avançadas da EPE-UNIFESP. Será composto por três eixos condutores, onze disciplinas e três linhas de pesquisas articuladas em torno deles.

A área de concentração Prática Avançada de Enfermagem na Saúde da Mulher foi estruturada para preparar enfermeiros para exercerem suas atividades com autonomia, dentro dos princípios da EPA, da prática baseada em evidência, da integralidade da saúde da mulher, do Código de Ética e de Deontologia da Enfermagem e diretrizes do Ministério da Saúde, da OMS e da OPAS, produzindo conhecimento inovador para solucionar problemas e melhorar os indicadores de saúde e bem-estar da mulher.

Os três eixos que darão sustentação para o alcance dos objetivos, das competências e das linhas e projetos de pesquisa congregam onze disciplinas e estão a seguir descritos.

O eixo Cuidado avançado no ciclo gravídico puerperal e neonatal, com ênfase na autonomia do enfermeiro é composto por quatro disciplinas que visam desenvolver conhecimento especializado, habilidades clínicas de alta performance, autonomia intelectual e postura investigadora, atualizada e crítica para a assistência às mulheres no ciclo gravídico-puerperal, em situaçôes de risco habitual ou diante dos agravos mais prevalentes, na atenção primária, hospitalar ou extra-hospitalar, de modo inovador.

Visa também desenvolver o aprofundamento na discussão de políticas públicas da saúde da mulher e do recém-nascido, enfocando o fazer mais autônomo do enfermeiro. Os títulos das disciplinas são: Bases fisiológicas e patológicas para a prática avançada de enfermagem no pré-natal, parto e pós-parto; Políticas e Diretrizes para a Saúde da Mulher e do Recém Nascido do Ministério da Saúde, da OPAS e da OMS relacionadas à gravidez, ao parto e ao puerpério; Enfermagem avançada sobre a parturição no domicílio, casas de parto, centros de parto (teórico-prática); Diretrizes do ICN e da International Confederation of Midwives (ICM) sobre prática Avançada em Enfermagem Obstétrica: aspectos legais, mercado de trabalho e capacitação.

O eixo Cuidado avançado na saúde sexual, reprodutiva, prevenção e cuidado à mulher vítima de violência doméstica e sexual é composto por quatro disciplinas que visam refletir sobre Políticas e Diretrizes para a Saúde da Mulher do Ministério da Saúde, da OPAS e da OMS relacionadas à sexualidade, ao gênero e à violência por meio de discussão crítica e reflexiva sobre a atuação do EPA na saúde sexual e reprodutiva da mulher, nas decisóes sobre concepção, na violência sexual e doméstica contra a mulher e na opção de gênero, problemas interligados e muito frequentes em nossa sociedade. Enfermeiros de prática avançada devem atuar nessas questôes de modo reflexivo, criativo e transformador, visando à promoção da saúde física e mental da mulher, a atuação que resguarde a dignidade, a segurança, a potência laboral e a qualidade de vida das mulheres.

Os títulos das disciplinas são: Políticas e Diretrizes para a Saúde da Mulher do Ministério da Saúde, da OPAS e da OMS relacionadas à sexualidade, ao gênero e à violência; Apoio emocional e aconselhamento a pessoas em situaçôes traumáticas; 
Enfermagem avançada em instituições que previnem, inibem e punem a violência contra a mulher; Enfermagem avançada na garantia dos direitos sexuais e reprodutivos.

O eixo Produção do conhecimento, desenvolvimento tecnológico e gestão em saúde é composto por três disciplinas que visam analisar princípios e práticas da epidemiologia de orientação clínica, o planejamento, a administração, a execução e a avaliação de desenhos de pesquisa e a avaliação de resultados de processos assistenciais. Pretende-se capacitar para o planejamento, o desenvolvimento, o teste e o registro de algoritmos decisórios, softwares e artefatos e competência para o uso de Tecnologia da Informação e Comunicação (TIC), visando atuação inovadora e transformadora da realidade. Os títulos das disciplinas são: Metodologia de pesquisa e epidemiologia clínica aplicadas à prática avançada de enfermagem na saúde da mulher; Fundamentos, métodos, processos e tecnologias em Enfermagem e Saúde e Ferramentas de qualidade aplicadas à Prática Avançada em Enfermagem.

As disciplinas visam dar sustentação aos eixos norteadores e às linhas de pesquisa e serão oito disciplinas teóricas e três teórico-práticas. As disciplinas teóricas e teórico-práticas permitirão o alcance das competências clínicas da EPA e as de um mestre.

A avaliação visa identificar o desenvolvimento de habilidades críticas e reflexivas do aluno, sua capacidade de incorporar novos conhecimentos, propor soluçôes criativas, inovadoras e transformadoras $\mathrm{da}$ realidade, consoante às políticas nacionais de saúde e à prática baseada em evidência. A avaliação será contínua e individual, tendo como base frequência, desempenho em provas, participação nos debates, atividades de campo, apresentação de seminários, resenhas críticas, resolução de problemas e elaboração de projeto de pesquisa com potencial transformador para a prática na saúde da mulher/ neonato. A nota será distribuída entre as estratégias de avaliação escolhidas.

As linhas de pesquisa visam nortear olhar investigativo sobre a realidade, nutrem-se e orientam o conjunto de disciplinas e são quatro: Enfermagem avançada no pré-natal, parto e pós-parto: melhoria da saúde e bem-estar; Enfermagem avançada na atenção à mulher nos aspectos da sexualidade, gênero e prevenção da violência doméstica e sexual: melhoria da saúde e bem-estar; Explorando as práticas avançadas do enfermeiro no cuidado crítico da mulher e seu filho de alto risco e Desenvolvimento e uso de tecnologia no cuidado à saúde da mulher e do neonato.

A disciplina Enfermagem avançada no pré-natal, parto e pós-parto de risco habitual e alto risco: melhoria da saúde e bem-estar compreende o desenvolvimento de estudos, projetos e programas inovadores e transformadores da realidade relativos ao cuidado avançado da mulher e seu filho no pré-natal, parto e pós-parto, visando aproximar os indicadores de saúde materno e neonatal brasileiros aos propostos pela OMS/OPAS e Ministério da Saúde do Brasil.

Já a disciplina Enfermagem avançada na atenção à mulher nos aspectos da sexualidade, gênero e prevenção da violência doméstica e sexual: melhoria da saúde e bem-estar compreende o desenvolvimento de estudos, projetos e programas inovadores e transformadores da realidade relativos ao cuidado da mulher no planejamento reprodutivo, no exercício da sua sexualidade e gênero e na prevenção e cuidado às vítimas de violência doméstica e sexual, visando aproximar os indicadores brasileiros aos propostos pela OMS/OPAS, Ministério da Saúde do Brasil e outras organizaçôes da sociedade civil, jurídicas, legislativas e governamentais.

A disciplina Desenvolvimento e uso de tecnologia no cuidado à saúde da mulher e neonato compreende estudos sobre o desenvolvimento, a avaliação tecnólogica, a implementação e as implicaçóes do uso de dispositivos, algorítmos de decisão, medicamentos, softwares e sistemas de organização e suporte, entre outros aparatos tecnológicos, para promover a saúde e o bem-estar da mulher e do neonato, visando a ação transformadora sobre indicadores de saúde.

\section{Duração do Programa, Créditos em disciplina, Processo seletivo e Trabalho de fim de curso (dissertação)}

O programa terá duração de 24 meses, exigirá o cumprimento de, no mínimo, 25 créditos em dis- 
ciplinas teóricas e teórico-práticas e elaboração de dissertação.

A dissertação deve ser vinculada à finalidade do mestrado profissional e à área de atuação do profissional-aluno, podendo ser de diversos formatos: estudos científicos, projetos de intervenção, construção e/ou implementação de protocolos assistenciais e gerenciais e desenvolvimento de produtos tecnológicos (softwares, algoritmos de decisão e artefatos, entre outros) relativos ao cuidado da mulher. $\mathrm{O}$ trabalho final deverá resultar de pesquisa e gerar um produto com características de EPA, inovador, capaz de responder às demandas sociais e de transformação da realidade.

O processo seletivo será feito por meio de prova de conhecimento sobre saúde da mulher e políticas públicas de saúde da mulher, a partir de referências bibliográficas previamente divulgadas; análise de projeto de pesquisa, dentro do escopo previsto no trabalho de fim de curso; análise de currículo; entrevista; e proficiência na língua inglesa.

Poderão candidatar-se enfermeiras obstetras, obstetrizes e enfermeiras de estratégia de saúde da família, enfermeiras de saúde pública e cuidado pré-natal, que deveráo atender a pré-requisitos. Como um dos objetivos é aumentar a autonomia dos enfermeiros no pré-natal, parto e puerpério, exige-se prévia formação e experiência nessa área. Aumentar a autonomia e segurança na tomada de decisão clínica refere-se a propiciar vivência do profissional em diversos cenários de parto e puerpério como casas de parto, centros de parto e parto em domícilio.

Enfermeiras obstetras e Obstetrizes deverão comprovar experiência em pré-natal e parto. Enfermeiras da Estratégia de Saúde da Família, Enfermeiras em Saúde Pública ou Enfermeiras na Atenção Básica deverão comprovar experiência na assistência pré-natal e cuidados pós-parto. Somente o profissional habilitado legalmente para a assistência ao parto executará as práticas relativas ao parto.

\section{Infraestrutura, recursos de informática e biblioteca}

Para o desenvolvimento de um programa de mestrado profissional é necessária adequada infra-estrutura que compreende área física para o desenvolvimento de aulas presencias, salas de estudo, salas de reunióes, salas e equipamentos de teleconferências, laboratórios de informática com disponibilidade de equipamentos para alunos e docentes, rede wi-fi e recursos multimídia para docentes e discentes, em salas de aula e outros espaços da instituição. Exige-se biblioteca física para a guarda e consulta do acervo e local de estudo. $\mathrm{O}$ acervo da biblioteca deve compor-se de obras da área da saúde, enfermagem e correlatas e relacionadas à pesquisa e análise de dados, com livros, revistas e dissertaçóes e teses, por meios físicos e eletrônicos e deve prover amplo acesso eletrônico ao material que disponibiliza aos alunos.

Há necessidade de laboratórios de ensino e pesquisa e parcerias com instituiçôes assistenciais relacionadas à saúde da mulher para prover as experiências clínicas necessárias para alcançar os objetivos do curso, bem como parcerias com instituiçóes envolvidas na inibição e punição da violência contra a mulher, contra a discriminação de gênero e de transexuais e de acolhimento e cuidado à mulher em situação traumática e de discriminação, também objetivos do curso.

\section{Discussão}

Frente à abrangência, à importância epidemiológica, às carências e às políticas do Ministério da Saúde, da OPAS e da OMS, que estimulam o enfrentamento aos desafios do cuidado à saúde da mulher, somada à inexistência de programa com enfoque em EPA, fica claro haver espaço para novos programas, especialmente aqueles voltados para o avanço na produção de conhecimento aplicado à área de saúde da mulher. O MEPASM visa desenvolver competências para intervir e transformar positivamente a realidade de saúde e produzir tecnologia condizentes às necessidades locais, como é a vocação do mestrado profissional e da EPA.

Parte significativa dos alunos de pós-graduação acadêmica é oriunda de ambientes de fora da academia e parcela deles busca, de fato, maior qualificação profissional, não propriamente tornar-se pesquisador, e cursam mestrado acadêmico pela ausência de opção de pós-graduação profissional. 
A proposta do MEPASM é trabalhar com profissionais atuantes na assistência aliando, de forma efetiva, a capacitação de recursos humanos para o exercício da prática profissional avançada e transformadora, com temática voltada ao desenvolvimento de pesquisa aplicada, demandada por setores externos à universidade. O programa quer acrescentar qualidade ao cuidado, construir e aperfeiçoar projetos de melhorias e políticas públicas que elevem os indicadores de saúde e bem-estar da mulher.

O ICN contribuiu grandemente com a evolução da EPA ao conceituar, especificar a formação, definir papéis e regulamentar a ação desse profissional, o que fortalece diretrizes para a evolução da profissão no mundo. Propóe também que a educação seja em nível avançado (mínimo de mestrado), que haja reconhecimento formal (acreditação) dos programas que preparam enfermeiros para práticas avançadas e sistema formal de licenciamento, registro e credenciamento do profissional. Preconiza também a harmonia entre formação, papéis e regulamentação desses profissionais com as normas legais de cada país. International Council of Nurses. ${ }^{(4)}$

As características da prática do EPA são: integração da clínica, pesquisa, educação e gestão; alto grau de autonomia profissional e prática independente; gestáo de caso; ter seus próprios pacientes; habilidades avançadas de avaliação, tomada de decisão e raciocínio diagnóstico; competências clínicas avançadas reconhecidas; consultoria a provedores de saúde; planejamento, implementação e avaliação de programas e reconhecimento pelos clientes como primeiro ponto de contato. ${ }^{(4)}$

Os mecanismos regulatórios do EPA, específicos de cada país são: direito de diagnosticar; autoridade para prescrever medicação; autoridade para prescrever tratamento; autoridade para encaminhar clientes para outros profissionais; legislação que confere e protege o título de EPA; legislação ou outra mecanismo regulatório específico para Enfermeiro de Prática Avançada e títulos oficialmente reconhecidos para os enfermeiros desenvolvendo papéis de prática avançada. ${ }^{(4)}$ No Brasil, enfermeiras obstetras têm autoridade para admitir e liberar pacientes em hospitais e autoridade para assinar a declaração de nascido vivo.
Os dois principais papéis de EPA são Clinical Nurse Specialist (CNS) e Nurse Practitioner (NP), ainda sem tradução entre nós. CNSs têm grande responsabilidade em atividades não clínicas, como educação das colegas e melhoria de qualidade do cuidado por meio da organização de protocolos, proposição de políticas etc. NP têm grande envolvimento no cuidado clínico (cuidado às mulheres), com autonomia e autoridade, com liberdade para pedir testes, fazer diagnósticos e prescrever tratamentos, inclusive medicaçóes. ${ }^{(9)}$ Enfermeiros obstetras de prática avançada poderão atuar à semelhança de CNS ou como NP, pois há espaço profissional e legislação que permitem o desempenho de ambos os papéis. O MEPASM foi organizado para impulsionar o exercício desses dois papéis.

O momento atual inspira a que, de modo coordenado, trabalhemos para ampliar o papel do enfermeiro no Brasil, aperfeiçoando o processo de formação, regulamentação e definição de papéis para a implementação de práticas avançadas. As áreas com maior prontidão para a implantação da EPA são a Enfermagem Obstétrica e a Enfermagem Comunitária (Atenção Primária), por terem formação clínica inicial padronizada, especialmente nos programas de residência, pelos papéis que já exercem e pelo amparo legal que a Lei do Exercício Profissional e o Ministério da Saúde provêm.

A Saúde da Mulher compreende açôes diversas e complexas em todos os ciclos de vida, que se estendem da prevenção à reabilitação de doenças, da promoção da saúde física e mental, da opção de concepção e maternidade, do livre exercício da sexualidade, da inserção da mulher no mercado de trabalho, da compreensão da construção social de gênero e da violência contra a mulher, entre outras. As mulheres representam mais da metade da população, são cerca da metade da força de trabalho e influenciam, majoritariamente, no cuidado da família e no preparo das novas geraçóes.

A ICM estabeleceu as competências essenciais e o conjunto de saberes, habilidades e comportamentos profissionais requeridos de uma enfermeira obstetra e esses requisitos são também preconizados pela OMS. O perfil propóe Competências gerais para as áreas Cuidado Pré-gravidez e Pré-natal, 
Cuidados durante o Parto e Nascimento e Cuidados Pós-parto e Neonatal. ${ }^{(10)}$

Espera-se que a Enfermeira Obstetra/Obstetriz seja capaz de: 1) assumir responsabilidade por suas decisóes e açóes com autonomia; 2) assumir responsabilidade pelo autocuidado e autodesenvolvimento como enfermeira obstetra/obstetriz; 3) delegar adequadamente aspectos do cuidado e supervisionar; 4) utilizar pesquisa para embasar a prática; 5) repeitar direitos fundamentais humanos ao prover cuidados obstétricos e de saúde da mulher; 6) obedecer às leis, aos regulamentos e aos códigos de conduta da enfermagem obstétrica; 7) facilitar a escolha da mulher sobre o seu cuidado; 8) demonstrar comunicação interpessoal efetiva com mulheres e família, equipes de cuidado em saúde e grupos comunitários; 9) facilitar o processo de parto normal em ambientes institucionais e comunitários, incluindo os domicílios; 10) avaliar o estado de saúde e riscos à saúde e promover o cuidado e o bem-estar de mulheres e neonatos; 11) prevenir e tratar problemas de saúde relacionados à reprodução e ao neonato; 12) reconhecer anormalidades e complicaçôes e instituir tratamento e encaminhamento adequados; 13) cuidado da mulher que experiencia abuso e violência física e sexual e 14) cuidado pré-gravidez e pré-natal. ${ }^{(10)}$

As enfermeiras obstetras brasileiras adequam-se às características descritas pela ICM, mas há lacunas. O MEPASM pretende incrementar: a autonomia profissional, a competência clínica, a responsabilidade pelo autodesenvolvimento contínuo, o hábito de embasar as condutas profissionais por pesquisas, o parto domiciliar em instituiçóes comunitárias e o cuidado à mulher que experiencia abuso e violência física e sexual.

A contribuição da enfermagem para o cuidado à saúde da mulher no Brasil já é muito significativa. No entanto, há diversos aspectos ainda não adequadamente atendidos como: o alto índice de cesárea, a alta morbi-mortalidade materna e neonatal precoces, o desmame precoce, o alto índice de gravidez na adolescência, a inadequada educação sexual e a interrupção da gravidez em situação de risco, os altos índices de violência obstétrica, doméstica e sexual contra a mulher, a discriminação das pessoas trans- sexuais, o climatério, o envelhecimento desassistido, os altos índices de transtornos de humor e ansiedade e o insuficiente conhecimento e implementação das políticas e diretrizes nacionais sobre a mulher, entre outros. Há diversos programas e protocolos nacionais e internacionais sobre essas questóes, ainda pouco explorados na academia e entre profissionais da clínica e, consequentemente, pouco adaptados e implantados em nosso meio.

Desse conjunto de lacunas existentes no cuidado à saúde da mulher, o MEPASM terá como eixo norteador aumentar a autonomia da enfermeira obstetra no pré-natal, parto e puerpério, visando humanizar as condiçóes de parto, diminuir o número de cesárias e diminuir as taxas de mortalidade pré-natal, dentro do conceito de melhores práticas em saúde. $\mathrm{O}$ segundo eixo norteador é a atenção qualificada à mulher vítima de violência, visando acolher, cuidar e empoderar a mulher nessa condição, nos diversos cenários de atendimento. $\mathrm{O}$ último eixo é a atenção emancipadora à saúde sexual e reprodutiva. O preparo de EPA para auxiliar a mulher no exercício do seu controle reprodutivo visa maior autonomia dos profissionais para aconselhar e indicar métodos contraceptivos, além de aumentar a competência da EPA para o atendimento de mulheres transexuais, no cuidado à sua saúde e no direito de vivenciar a sua sexualidade.

$\mathrm{O}$ conceito EPA do ICN aplica-se também à Enfermagem na Saúde da Mulher. Estudo recente destacou como atributos desse profissional a autonomia na prática, a liderança, a expertise e as habilidades de consumo de pesquisas. Como papéis profissionais para EPA em Saúde da Mulher foram apontados: enfermeiras clínicas (clinicians), líderes clínicas e professionais, educadoras, consultoras, gerentes, agentes de mudança, pesquisadoras e auditoras. ${ }^{(11)}$

Os atributos e papéis do MEPASM estão em acordo ao descrito na definição do ICN sobre EPA, a saber, profissionais com habilidades e competência clínica para tomar decisóes complexas, expandir e aperfeiçoar a prática de enfermagem, promover maior inclusão e melhor cuidado na assistência à saúde. Nota-se que, no Brasil, já temos enfermeiras e enfermeiras obstetras exercendo alguns desses pa- 
péis. No entanto, a formação para eles não é padronizada, nem sempre há reconhecimento profissional e financeiro e nem sempre quem o exerce, tem de fato competência para esse fim. Urge organizar o sistema de EPA no Brasil.

A autonomia é central para a EPA. A prática autônoma em EPA significa que o profissional é responsável e respeitado por sua prática; que é capaz de prover cuidado para a mulher e sua família de modo autônomo e que o processo de decisão é independente e vai além do manejo obstétrico. ${ }^{(11)}$

A liderança do EPA pode ser clínica ou gerencial. A liderança clínica refere-se a dar suporte para o aperfeiçoamento da prática clínica nos serviços; inclui a orientação e a coordenação de grupos multiprofissionais, a atuação como agente iniciador de mudança no cuidado ao doente, assumir responsabilidade por políticas, desenvolvimento e implementaçáo de guias e protocolos, programas educacionais formais, treinamento em serviço e cursos de atualização para grupos multiprofissionais com enfoque nas mudanças da prática clínica. EPA atuam como preceptores para colegas e alunos que, encorajando o aprendizado, servem como modelo para a tomada de decisão com autonomia e desenvolvimento profissional. A liderança gerencial de EPA refere-se às atividades de ensino, ao desenvolvimento de políticas e ao engajamento em comitês profissionais que extrapolam o serviço, alcançando âmbito nacional e internacional. Pode haver envolvimento no desenvolvimento de currículo e requer a consciência política sobre os fatores que afetam o cuidado e a profissão. ${ }^{(11)}$

A expertise é atributo fundamental da EPA e significa conhecimento profundo, amplo (não pode ser restrito, confinado a uma área muito específica) e experiência prática sólida. ${ }^{(11)}$

A habilidade de pesquisa é parte das competências da EPA e pode ser o desenvolvimento de pesquisas próprias, a colaboração com outros pesquisadores ou grupos de pesquisa, ser consumidor de pesquisa ou fazer a translação da pesquisa para a prática. ${ }^{(11)}$

A análise das disciplinas e linhas de pesquisa previstas no MEPASM mostra que foram intencionamente organizadas para auxiliar os alunos a adquirirem os atributos de um EPA e de um mestre e prepará-los para desempenhar os papéis a eles reservados. Observam-se disciplinas e linha de pesquisa sobre o cuidado clínico avançado, sobre programas e políticas de saúde, preparação para a pesquisa e uso e desenvolvimento de tecnologia, abarcando os três eixos do programa.

Disciplinas téorico-práticas são fundamentais em mestrado profissional tendo a EPA como fim, mas não em programas de mestrado acadêmico. No mestrado profissional, quer-se que o aluno incorpore novas habilidades clínicas, desenvolva capacidade de reflexão, tomada de decisóes complexas e maior autonomia para atuar em situaçôes de pré-parto, parto e puerpério em casas de parto, centros de parto e domicílio. Quer-se também aperfeiçoar a atuação junto à mulher vítima de violência e nas questóes de gênero. $\mathrm{O}$ aprimoramento dessas competências requer vivência e reflexão crítica em cenários não usuais na formação. A insuficiente compreensão das diferenças de objetivos e processos formativos do mestrado profissional e acadêmico tem resultado que muitos programas de mestrado profissional são, na verdade, mestrado acadêmico.

O caráter científico e tecnológico de um mestre profissional exige desenvoltura para analisar a realidade e implementar políticas públicas, organizar e implementar protocolos clínicos e propor soluçóes tecnológicas, tendo como meta a atuação transformadora da realidade, aderente aos modelos de prática avançada em enfermagem e da prática baseada em evidência.

\section{Conclusão}

Há oportunidades para se aperfeiçoar o cuidado à mulher, em muitos aspectos, como aumentando a autonomia do enfermeiro obstetra para a atuação no pré-natal, parto e puerpério, ampliando suas competências no combate à violência doméstica e sexual contra a mulher e ampliando a qualificação do profissional para o cuidado que contribua com a mulher no livre exercício da sua sexualidade e identidade de gênero. $\mathrm{O}$ exercício dessas novas áreas, se feitos dentro dos preceitos de autonomia, alta competência clínica, liderança qualificada e habilidades 
de pesquisa, serão transformadores da realidade e caracterizarão o enfermeiro, que expandirá a sua atuação além daquela prevista na formação inicial, isto é, caracterizará a EPA. Espera-se que a organização do Mestrado Profissional em Prática Avançada em Enfermagem na Saúde da Mulher (MEPASM) contribua para divulgar, entre nós, a ideia do que é EPA, estimule a proposição de outros programas pela academia, a atuação dos órgãos de classe profissional no ajuste da legislação e da regulamentação desses novos papéis e a incorporação desses profissionais no mercado de trabalho.

\section{Referências}

1. The All-Party Parliamentary Group on Global Health (APPG-Global Health). 2018-2019 Annual Report and Accounts. 27th June 2018 - 26th June 2019. [cited on 3 Mar 2020]. APPG-Global Health; 2018/2019. Available from: http://www.appg-globalhealth.org.uk download/i/mark_dl/u/4009611296/4636821095/APPG-GH\%20 Annual\%20Report\%20and\%20accounts\%202018-19\%20-\%20 JR\%2023\%20Aug\%202019.pdf

2. Coordenação de Aperfeiçoamento de Pessoal de Nível Superior (CAPES). Mestrado e Doutorado, o que são? Brasília (DF): CAPES; 2014 [citado 2020 Mar 3] Disponível em: https://www.capes.gov.br/ avaliacao/sobre-a-avaliacao/mestrado-e-doutorado-o-que-sao

3. OPAS. Organização Pan-Americana da Saúde. Ampliação do papel dos enfermeiros na atenção primária à saúde. Washington (D.C.): OPAS; 2018. 54 p.
4. International Council of Nurses (ICN). ICN Advanced practice nursing network. definition and characteristics of the role. ICN; c2001. [cited 2020 Mar 3]. Available from: https://international.aanp.org/Practice/ APNRoles

5. Royal College of Nursing (RCN). Advanced Practices Standards. RCN standards for advanced level nursing practice. RCN; c2020. [cited 2020 Mar 3]. Available from: https://www.rcn.org.uk/professionaldevelopment/advanced-practice-standards

6. 2020: unleashing the full potential of nursing. Lancet. 2019;394(10212):1879.

7. Conselho Federal de Enfermagem (COFEN). Para OMS, Saúde universal exige práticas avançadas de Enfermagem. Brasília (DF):COFEN; 2018. [citado 2020 Mar 3]. Disponível em: http://www. cofen.gov.br/para-oms-saude-universal-exige-praticas-avancadasde-enfermagem_62722.html

8. Brasil. Ministério da Educação. Portaria Normativa No. 7 de 22 de junho de 2009. Dispõe sobre o mestrado profissional no âmbito da Fundação Coordenação de Aperfeiçoamento de Pessoal de Nível Superior - CAPES. Brasília (DF): Ministério da Educação; 2009. [citado 2020 Mar 3]. Disponível em: https://www.unifesp.br/campus/sao/ meparem/images/docs/Portaria_Normativa_N_07_de_22_de_ junho_de_2009.pdf

9. Bryant-Lukosius D, Martin-Misener R. ICN Policy Brief. Advanced practice nursing: na essential component of country level human resources for health. Geneva: WHO. s/d. [cited 2020 Mar 3]. Available from: https://www.who.int/workforcealliance/knowledge/resources/ ICN_PolicyBrief6AdvancedPracticeNursing.pdf?ua=1

10. International Confederation of Midwives (ICN). Essential Competencies for Midwifery Practice 2019 Update. ICN; 2019. [cited 2020 Mar 3]. Available from: https://www.internationalmidwives.org/assets/files/ general-files/2019/10/icm-competencies-en-print-october-2019_ final_18-oct-5db05248843e8.pdf

11. Goemaes R, Beeckman D, Goossens J, Shawe J, Verhaeghe S, Van Hecke A. Advanced midwifery practice: an evolutionary concept analysis. Midwifery. 2016;42:29-37. 